\title{
MONITOREO DE LA POBLACIÓN DE PERROS FERALES EN LA ISLA DE CEDROS, BAJA CALIFORNIA, Y LAS AMENAZAS A LA MASTOFAUNA NATIVA
}

\author{
MARÍA CONCEPCIÓN GARCÍA-AGUILAR \\ Lab. de Ecología de Pinnípedos, Centro Interdisciplinario de Ciencias Marinas del Instituto Politécnico \\ Nacional. Av. IPN s/n, Col. Playa Palo de Santa Rita, CP 23096, La Paz, B. C. S., México. \\ Dirección actual: Depto. de Biología de la Conservación, Centro de Investigación Científica y de \\ Educación Superior de Ensenada. Carretera Tijuana-Ensenada Num. 3918, CP 22860, \\ Ensenada, B. C., México. \\ <gaguilar@cicese.mx>; <mcgarcia2002@yahoo.com>
}

García-Aguilar, M. C. 2012. Monitoreo de la población de perros ferales en la Isla de Cedros, Baja California, y las amenazas a la mastofauna nativa. Acta Zoológica Mexicana (n. s.), 28(1): 37-48.

RESUMEN. El monitoreo de especies introducidas es una herramienta importante para preservar las especies nativas. El objetivo de este estudio fue monitorear a la población de perros ferales en la Isla de Cedros e identificar las amenazas actuales y potenciales de su presencia sobre la mastofauna nativa. La colecta de datos se realizó en dos áreas de la isla, la costa noreste (CNE) y la región centro-sur (RCS). Los patrones de distribución y la densidad de los perros se determinaron con un método no invasivo basado en el conteo de excretas a lo largo de transectos y se estimó la densidad relativa (R) y el índice de abundancia relativa fecal (fRAI). La composición de la dieta se evaluó por medio del análisis de las excretas. Tanto los valores de R como de fRAI fueron significativamente mayores $(\mathrm{P}<0.05)$ en $\mathrm{CNE}$ que en RCS. Los resultados mostraron que los perros se alimentan de al menos tres especies de mamíferos endémicos (el venado bura, el conejo matorralero y el ratón de abazones de Cedros) y de dos especies de pinnípedos (el elefante marino del norte y el lobo marino de California). A partir de este estudio se cuenta con una línea de base que podrá ser usada en los programas de control y monitoreo futuros. Sin embargo, es necesario desarrollar estudios sobre el comportamiento alimentario de los perros para evaluar su actividad predatoria y medir el nivel de daño asociado; además, dado que las interacciones entre pinnípedos y perros parecen ser frecuentes y están relacionados filogenéticamente, posiblemente la mayor amenaza de la presencia de los perros sea la propagación de enfermedades infeccionas, con graves resultados para la vida silvestre.

Palabras clave: perros ferales, áreas insulares, mastofauna, amenazas.

García-Aguilar, M. C. 2012. Monitoring feral dog's population at Isla de Cedros, Baja California, and the associated threats over native mastofauna. Acta Zoológica Mexicana (n. s.), 28(1): 37-48.

ABSTRACT. Monitoring introduced species is an important management tool in order to preserve native species. The aim of this study was to monitor the feral dog's population in Isla de Cedros and to

Recibido: 18/10/2010; aceptado: 10/11/2011. 
identify actual and potential threats of their presence over the native mastofauna. Data were collected at two sampling areas of the island, the Northeast Coast (CNE) and the Central-South Region (RCS). Dog's distribution pattern and density was assess using a non-invasive method based on scat counts along transect surveys and relative density $(\mathrm{R})$ and faecal relative abundance index (fRAI) were estimated. Dog's diet composition was evaluated by scat analysis. Both R and fRAI were significant greater $(\mathrm{P}<0.05)$ in the CNE than in the RCS. The results showed that feral dogs are feeding from at least three endemic terrestrial mammal species (the mule deer, the brush rabbit, and the Anthony's pocket mouse) and two pinnipeds species (the northern elephant seal and the California sea lion). With this study provide a useful baseline for future comparison and monitoring and control purposes. Nevertheless, it is necessary to conduct studies about the dog's feeding behavior to measure their predatory activity and to assess the associated damage level. Also, due to the fact that interactions between dogs and pinnipeds appeared frequently and because they are phylogenetically related, the greatest threat of the dog's presence is that it could be a disease vector, with serious health outcomes for the wildlife.

Key words: Feral dogs, insular areas, mastofauna, wildlife threats.

\section{INTRODUCCIÓN}

El monitoreo de las poblaciones de las especies introducidas es un componente esencial en desarrollo de programas integrales de control: su seguimiento en el tiempo y el espacio puede ser utilizado como un predictor del nivel de daño en las áreas naturales, indicando cuándo el control es necesario e identificando las medidas más viables para la erradicación (Engeman \& Witmer 2000). Los perros ferales (Canis lupus familiaris) son los cánidos silvestres más ampliamente distribuidos en todo el mundo y su presencia en áreas naturales puede producir impactos ecológicos complejos, influenciando potencialmente a las comunidades nativas e incluyendo efectos indirectos que pueden propagarse como cascada, afectando plantas, animales y procesos ecológicos (Kay 1998; Smith et al. 2003). Los ecosistemas insulares son particularmente vulnerables al impacto de estos animales debido a que las especies nativas tienen pocas defensas, tanto fisiológicas como conductuales, ante la amenaza de un mamífero depredador introducido en el ecosistema. La depredación es la amenaza más evidente, pero los perros pueden ser un vector en la dispersión de enfermedades infecciosas y su presencia puede desplazar a algunas especies fuera de sus hábitats naturales.

La Isla de Cedros es la más grande del Pacífico Norte mexicano y sus variadas especies y subespecies endémicas de plantas y vertebrados la convierten en una importante área biológica. Los tipos de vegetación son el chaparral, el matorral costero, el bosque de pinos juníperos y el matorral de dunas (Morán 1972; Rzedowski 1978). En la isla habitan varias especies y subespecies de vertebrados endémicos (Mellink 1993). Hay tres especies endémicas de reptiles, dos lagartijas (Phrynosoma cerroense y Elgaria cedrosensis) y una víbora de cascabel (Crotalus exsul), y dos especies de roedores, el ratón de abazones de Cedros (Chaetodipus anthonyi) y la rata cambalachera de Cedros (Neotoma bryanti). Las subespecies endémicas de mamíferos incluyen al conejo matorralero (Sylvilagus bachmani cerrosensis), un ratón de cactus (Peromyscus eremicus cedrosensis) y el venado bura (Odocoileus hemionus cerro- 
sensis). Además, tres especies de pinnípedos habitan en las costas: el lobo marino de California (Zalophus californianus californianus), el elefante marino del norte (Mirounga angustirostris), y la foca común (Phoca vitulina richardsii).

La presencia de perros ferales en la Isla de Cedros fue reportada hace más de 15 años (Mellink 1993). Es la única especie depredadora de mamífero e información preliminar mostró que su dieta es muy variada e incluye mamíferos, aves y reptiles (Gallo-Reynoso \& García-Aguilar 2008). Sin embargo, hasta el momento no existen datos sobre la distribución y abundancia de los perros ni sobre los efectos de su presencia sobre la vida silvestre nativa. El objetivo de este estudio fue evaluar la distribución y densidad de la población de perros ferales en la Isla de Cedros e identificar sus presas principales, en particular las especies de mastofauna. La información resultante podrá ser empleada en programas de monitoreo a largo plazo y en la selección de las medidas de control, maximizando su eficiencia y minimizando los impactos sobre las especies nativas.

\section{MATERIAL Y MÉTODOS}

La Isla de Cedros se encuentra dentro del polígono del ANP "Valle de los Cirios" y se localiza en la región central de la costa occidental de la Península de Baja California, entre los $28^{\circ} 02^{\prime} 20^{\prime \prime}$ y $28^{\circ} 22^{\prime} 55^{\prime \prime} \mathrm{N}$ y $\operatorname{los} 115^{\circ} 09^{\prime} 20^{\prime \prime}$ y $115^{\circ} 21^{\prime} 30^{\prime \prime} \mathrm{W}$ (Fig. 1). Su longitud máxima es de $34 \mathrm{~km}$, con área aproximada es de $360 \mathrm{~km}^{2}$ y se caracteriza por presentar terreno escarpado de pendientes pronunciadas (Mellink 1993).

El trabajo de campo se realizó durante febrero del 2010 en dos áreas de la isla: la costa noreste (CNE) y la región centro-sur (RCS) (Fig. 1, Cuadro 1). Estas dos áreas representan una gran variación en las características ambientales y dada la longitud de la isla se consideraron independientes. Las áreas fueron muestreadas a través de transectos, que siguieron las características lineales (como arroyos y cañones); el ancho de cada transecto fue de 5-10 m. Las rutas de los transectos fueron recorridas por dos personas una sola vez y georreferidas con GPS (Magellan Triton 400), y los rastros (excretas, huellas y restos) de mamíferos fueron registrados. Para calibrar representativamente la abundancia de las excretas, se aplicó un factor de corrección de terreno rugoso $(\mathrm{Ln}=$ longitud del transecto $\times 1.3)($ Khorozyan 2003). Las excretas de los perros fueron colectadas de manera individual en bolsas plásticas herméticas y su posición fue registrada con GPS. Con base en color, dureza y textura, las excretas se clasificaron como recientes (tiempo de deposición $\leq 3 \mathrm{~d}$ ) y secas (tiempo de deposición $>3 \mathrm{~d})$.

La densidad relativa (R) de los perros se calculó como $R=\frac{s_{i}}{L n \times D}$ (Sadler et al. 2004), donde $s_{i}$ es el número de excretas recientes colectadas en el transecto $i, L n$ representa la longitud corregida del transecto y $D$ es el tiempo máximo de deposición 

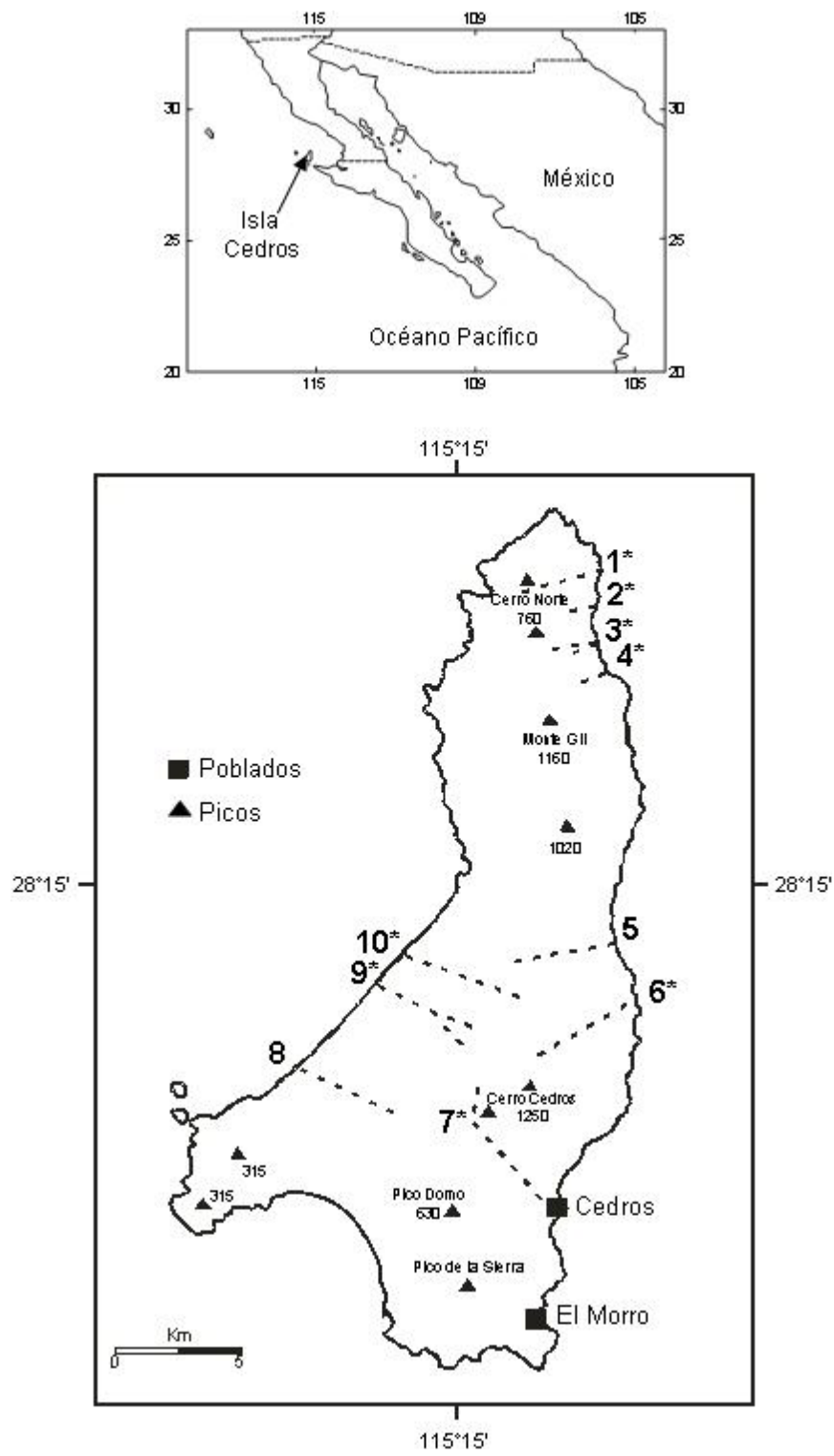

Figura 1. Isla de Cedros, Baja California. Las líneas punteadas representan los transectos recorridos durante febrero de 2010: 1) Arroyo del Faro, 2) Manantial de Punta Norte, 3) Dos Arroyos Norte y Dos Arroyos Sur, 4) La Lobera, 5) El Choyal, 6) El Gran Cañón, 7) Cerro Cedros, 8) Arroyo Los Pinitos, 9) Arroyo Los Tenis, y 10) Arroyo Vargas. El símbolo* señala los transectos donde se encontraron excretas de perros. 
Cuadro 1. Transectos recorridos en febrero de 2010 en la Isla de Cedros, Baja California, y número de excretas de perro colectadas. $\mathrm{CNE}=$ costa noreste, $\mathrm{RCS}=$ región centro-sur, $\mathrm{Lt}=$ longitud del transecto, $\mathrm{Ln}=\mathrm{Lt} \times 1.3$.

\begin{tabular}{llcccc}
\hline & & & \multicolumn{2}{c}{ Excretas } \\
\hline \multicolumn{1}{c}{ Área } & \multicolumn{1}{c}{ Transecto } & Lt $(\mathrm{km})$ & Ln $(\mathrm{km})$ & Total & Recientes \\
\hline CNE & Arroyo del Faro & 2.45 & 3.19 & 6 & 0 \\
& Manantial de Punta Norte & 1.16 & 1.51 & 9 & 5 \\
& Dos Arroyos Norte & 1.36 & 1.77 & 5 & 0 \\
& Dos Arroyos Sur & 0.92 & 1.19 & 9 & 2 \\
& Arroyo de La Lobera & 0.32 & 0.42 & 7 & 1 \\
Subtotal & & 6.21 & 8.08 & 36 & 8 \\
RCS & El Choyal & 3.20 & 4.16 & 0 & 0 \\
& El Gran Cañón & 3.36 & 4.37 & 4 & 0 \\
& Cerro Cedros & 6.81 & 8.86 & 1 & 0 \\
& Arroyo Los Pinitos & 3.19 & 4.14 & 0 & 0 \\
& Arroyo Los Tenis & 3.68 & 4.79 & 1 & 0 \\
Subtotal & Arroyo Vargas & 3.73 & 4.85 & 2 & 2 \\
& & 23.97 & 31.17 & 8 & 2 \\
\hline
\end{tabular}

$(D=3)$. El índice de abundancia relativa fecal (fRAI) se calculó como una medida indirecta de la abundancia relativa de los perros y se calculó como $\mathrm{fRAI}=$ número de excretas por $10 \mathrm{~km}$ de transectos caminados (Karanth et al. 2003; Khorozyan 2003).

Para el análisis de la dieta, las excretas fueron procesadas siguiendo protocolos establecidos (Reynolds \& Aebischer, 1991). Las fracciones macroscópicas se separaron tamizando las excretas (tamiz de ancho de malla $0.5 \mathrm{~mm}$ ) y los restos animales se clasificaron para su identificación. Los pelos obtenidos fueron analizados usando sus características internas (médula) y las marcas cuticulares (Terrink 1991). Para la identificación a nivel de especies, los pelos fueron comparados con material de referencia en el Laboratorio de Fauna Silvestre del Centro de Investigación Científica y de Educación Superior de Ensenada (CICESE) y con bibliografía especializada (Moore et al. 1974; Rodríguez de la Gala 2002). Se calculó la frecuencia de aparición (FA) de una presa como frecuencia total de la presa dividida entre el número total de excretas y el porcentaje de aparición (PA) como la frecuencia de la presa entre la suma de todas las frecuencias (Maher \& Brady 1986). Se comparó la diversidad de dieta entre áreas de muestreo (CNE vs. RCS) usando el índice de diversidad de Shannon-Weiner (Brower \& Zar 1984; Zar 1996). 


\section{RESULTADOS}

Se recorrieron $30.19 \mathrm{~km}(\mathrm{Ln}=39.24 \mathrm{~km})$ en 11 transectos (la longitud de los transectos varió en función de las características de terreno) (Cuadro 1; Fig. 1). Se encontraron rastros de venado bura (huesos, huellas y excretas) en 3 transectos (Dos Arroyos Norte, Arroyo de La Lobera y El Choyal) y de conejo matorralero (huellas y excretas) en 5 transectos (Arroyo del Faro, El Choyal, Arroyo Vargas, Arroyo Los Tenis y Arroyo Los Pinitos). En Dos Arroyos Sur se encontraron dos cadáveres de crías pinnípedos (uno lobo marino de California y otro elefante marino del norte) con evidencias de haber sido devorados por perros.

Se colectaron 44 excretas en 9 de los 11 transectos, de las cuales el $23 \%$ fueron recientes y se encontraron en 4 transectos: 3 localizados en CNE y uno en RCS (Cuadro 1). Los valores de la densidad relativa (R) fueron mayores en CNE que en RCS $(Z=-2.22$, g. $1 .=9, p<0.05)$ y lo mismo se observó al comparar los valores de fRAI entre las zonas de muestreo $(Z=2.74$ usando el total de excretas y $Z=2.46$ usando únicamente las excretas recientes, ambas con g. $1 .=9$ y $p<0.05)$ (Cuadro 2).

Con base en los restos encontrados se identificaron 10 tipos de presas (Cuadro 3) que se clasificaron en 3 grupos: mamíferos, aves y otros, donde se incluyeron una especie de invertebrado (langosta), un pez (posible género Raja) y plantas (Cuadro 4). La composición de la dieta de los perros fue diferente entre CNE y RCS, tanto a nivel de grupo $\left(\chi^{2}=14.91\right.$, g. $\left.1 .=2, p<0.01\right)$ como a nivel de especies $\left(\chi^{2}=64.04\right.$, g.

Cuadro 2. Densidad relativa de los perros (R) y valores del índice de abundancia relativa fecal (fRAI) en las dos áreas muestreadas de la Isla de Cedros. $\mathrm{CNE}=$ costa noreste, $\mathrm{RCS}=$ región centro-sur.

\begin{tabular}{llccc}
\hline & & $\mathrm{R}$ & \multicolumn{2}{c}{ fRAI } \\
\hline \multicolumn{1}{c}{ Área } & \multicolumn{1}{c}{ Transecto } & Excretas recientes & Total excretas & Excretas recientes \\
\hline CNE & Arroyo del Faro & 0.00 & 18.82 & 0.00 \\
& Manantial de Punta Norte & 1.10 & 59.63 & 33.13 \\
& Dos Arroyos Norte & 0.00 & 28.22 & 0.00 \\
& Dos Arroyos Sur & 0.56 & 75.66 & 16.81 \\
& Arroyo de La Lobera & 0.80 & 168.27 & 24.04 \\
Rubtotal & & 0.33 & 44.58 & 9.91 \\
& & 0.00 & 0.00 & 0.00 \\
& El Choyal & 0.00 & 9.16 & 0.00 \\
& El Gran Cañón & 0.00 & 1.13 & 0.00 \\
& Cerro Cedros & 0.00 & 0.00 & 0.00 \\
& Arroyo Los Pinitos & 0.00 & 2.09 & 0.00 \\
& Arroyo Los Tenis & 0.14 & 4.13 & 4.13 \\
& Arroyo Vargas & 0.02 & 2.57 & 0.64 \\
\hline
\end{tabular}


Cuadro 3. Restos encontrados en las excretas de los perros y usados para la identificación de las presas.

\begin{tabular}{lcccc}
\hline \multicolumn{1}{c}{ Presa } & Pelo & Plumas & Restos duros & Otros \\
\hline MAMÍFEROS & & & & \\
Mirounga aungustirostris & $\mathrm{X}$ & & $\mathrm{X}$ & Vibrisas \\
Zalophus californianus & $\mathrm{X}$ & & $\mathrm{X}$ & \\
Chaetodipus anthonyi & $\mathrm{X}$ & & $\mathrm{X}$ & \\
Roedores (no identificados) & $\mathrm{X}$ & & $\mathrm{X}$ & \\
Sylvilagus bachmani cerrosensis & $\mathrm{X}$ & & & \\
Odocoileus hemionus cerrosensis & $\mathrm{X}$ & & & \\
AVES (no identificados) & & $\mathrm{X}$ & & \\
OTROS & & & $\mathrm{X}$ & \\
Peces & & $\mathrm{X}$ & Semillas \\
Invertebrados & & & & \\
Plantas & & & & \\
\hline
\end{tabular}

Cuadro 4. Composición de la dieta de los perros en la costa noreste y la región centro-sur de la Isla de Cedros. FA = frecuencia de aparición, $\mathrm{PA}=$ porcentaje de aparición.

\begin{tabular}{lcccc}
\hline & \multicolumn{2}{c}{ Costa noreste } & \multicolumn{2}{c}{ Región centro-sur } \\
\cline { 2 - 5 } & FA & PA & FA & PA \\
\hline MAMÍFEROS & & 82.69 & & 66.67 \\
Mirounga aungustirostris & 75.00 & 51.92 & 0.00 & 0.00 \\
Zalophus californianus & 8.33 & 5.77 & 0.00 & 0.00 \\
Chaetodipus anthonyi & 13.89 & 9.62 & 0.00 & 0.00 \\
Roedores (no identificados) & 13.89 & 9.62 & 62.50 & 33.33 \\
Sylvilagus bachmani cerrosensis & 2.78 & 1.92 & 62.50 & 33.33 \\
Odocoileus hemionus cerrosensis & 5.56 & 3.85 & 0.00 & 0.00 \\
AVES (no identificados) & 13.89 & 9.62 & 50.00 & 26.67 \\
OTROS & & 7.79 & & 6.67 \\
Peces & & 1.92 & 0.00 & 0.00 \\
Invertebrados & 2.78 & 5.77 & 0.00 & 0.00 \\
Plantas (no identificadas) $_{\text {Total }}$ & 8.33 & 0.00 & 12.50 & 6.67 \\
\hline
\end{tabular}

a posible género Raja.

blangosta 
$1 .=9, p<0.01)$. Sin embargo, los mamíferos aparecieron como el grupo más importante en la dieta (Cuadro 4). En CNE las presas más comunes fueron los pinnípedos (M. angustirostris y Z. californianus) y los roedores (C. anthonyi y otras especies no identificadas); en RCS las presas más comunes fueron los roedores (no identificados) y el conejo matorralero (S. b. cerrosensis). La diversidad de la dieta fue significativamente mayor en $\mathrm{CNE}\left(\mathrm{H}^{\prime}=0.67\right)$ que en $\mathrm{RCS}\left(\mathrm{H}^{\prime}=0.55\right)(\mathrm{t}=1.99, \mathrm{~g} .1 .=63, p<$ $0.05)$.

\section{DISCUSIÓN}

Para el desarrollo de programas de monitoreo y control de especies introducidas es posible diseñar métodos de campo que, con las apropiadas restricciones en tiempo y espacio, pueden proveer la información necesaria para la toma de decisiones de manejo (Caughley 1977; Engeman \& Allen 2000). En este estudio se usó un método no invasivo basado en el conteo de excretas para evaluar los patrones de distribución y la densidad de la población de perros ferales en la Isla de Cedros y se analizó la composición de su dieta. El método es simple y de fácil aplicación en el campo, pero con la sensibilidad suficiente para reflejar los cambios en la población. Los resultados sugieren que los perros ferales se distribuyen principalmente en la región más norteña de la isla. Los mamíferos aparecen como el grupo más importante de su dieta, pero la composición fue diferente entre áreas: en el norte de la isla los pinnípedos fueron las presas más comunes, mientras que en el centro lo fueron los roedores y el conejo.

Los métodos indirectos de campo (conteo de rastros) han sido utilizados para medir las variaciones espaciales y temporales en la densidad animal (Wilson \& Delahay 2001; Sadler et al. 2004). Estos métodos son de gran utilidad en estudios a largo plazo porque su aplicación en campo es relativamente simple y sus costos reducidos; además, los rastros suelen ser detectables a lo largo de la distribución de los animales y en diferentes tipos de hábitat (Jachmann 1991). Los perros ferales habitan en áreas donde el acceso humano es limitado y en sitios remotos donde se alimentan de especies nativas (Green \& Gipson 1994). La evaluación de sus poblaciones puede ser complicada debido a que sus poblaciones son relativamente escasas, que poseen ámbitos hogareños grandes, su comportamiento es evasivo y habitan en terrenos de difícil acceso. Por ello, el conteo de excretas es un método adecuado para detectar su presencia y estimar su densidad (Sutherland 1996).

El muestro de excretas se usa para estimar densidad, evaluar los movimientos de los animales, en estudios de alimentación y enfermedades, así como en análisis de ADN y hormonales (Cavalli 1994; Sharp et al. 2001; Wasser et al. 2004; De Luca \& Mpunga 2005; Mitchell \& Balogh 2007; Furtado et al. 2008). Sin embargo, el uso del conteo de excretas para estimar densidad ha sido criticado debido a las diferencias en la persistencia de las excretas por cuestiones ambientales y climáticas y por el error de observación (Webbon et al. 2004). Para minimizar estas fuentes de error, 
en este estudio se utilizaron en el análisis de la distribución y densidad únicamente excretas recientes colectadas durante una misma temporada (invierno), con lo cual se redujo el efecto de los patrones estacionales en la tasa de deposición y de los eventos climáticos.

Estudios realizados con perros salvajes (e. g. dingos) han mostrado que el ámbito hogareño varía de 10 a $90 \mathrm{~km}^{2}$, dependiendo del tipo de hábitat y la disponibilidad de alimento (Fleming et al. 2001). Aproximadamente el 10\% del área que cubre el ámbito hogareño es usada de manera frecuente en los movimientos de búsqueda (movimientos asociados a la cacería y caracterizados por una actividad intensa en áreas pequeñas y siguiendo patrones bien definidos a lo largo de características topográficas); el restante $90 \%$ del área es utilizada en los movimientos exploratorios (movimientos entre áreas de cacería y alrededor de los límites del ámbito hogareño) (Corbett 2001; Mitchell \& Balogh 2007). Se encontraron excretas en todos los transectos recorridos en la costa noreste de la isla, pero las excretas recientes fueron localizadas solamente en 3 de ellos. Dado el tamaño del ámbito hogareño, es posible que un solo grupo de perros habite en la costa noreste, moviéndose sin restricciones desde La Lobera hasta la Punta Norte. Una situación similar podría estar sucediendo en la costa oeste de la isla (Arroyo Vargas, Arroyo Los Tenis y Arroyo Los Pinitos), donde las excretas recientes solo se encontraron en un transecto pero en todos se registraron huellas. Por otra parte, la colecta sistemática de excretas a lo largo de transectos establecidos puede proporcionar suficiente información sobre la variación de la abundancia relativa. Los resultados tanto de la estimación de la densidad relativa como los valores del fRAI mostraron que la abundancia de perros es mayor en la costa noreste que en la región centro-sur de la isla.

Los perros ferales son consumidores oportunistas: pueden ser eficientes depredadores o carroñeros (Green \& Gipson 1994); además, pueden alimentarse de gran variedad de presas, pero son relativamente pocas especies las que constituyen el grueso de su dieta (Mitchell \& Banks 2005). Los mamíferos fueron el grupo más importante en la dieta de los perros de la Isla de Cedros, tanto en la costa noreste (el elefante marino del norte) como en la región centro-sur (roedores y conejo), pero la diversidad fue mayor en la costa noreste. Estas diferencias en la composición y diversidad de la dieta están influenciadas por la disponibilidad de presas, pero también sugieren que posiblemente no exista intercambio de animales entre las dos áreas muestreadas, soportando la idea del ámbito hogareño planteada en el párrafo anterior.

Las colonias de elefante marino del norte y de lobo marino de California se localizan en la costa noreste de la isla (Stewart et al. 1994; Lowry \& Maravilla-Chávez 2005), siendo las presas principales para los perros de esta área. No existe información sobre la distribución y abundancia de los mamíferos terrestres nativos, pero con base en los resultados obtenidos en este estudio es posible hacer algunas inferencias: 1) la distribución de la subespecie endémica de venado bura probablemente esté res- 
tringida a la región norte de la isla; 2) aparentemente, la distribución de la subespecie endémica de conejo matorralero es más amplia, pero podría ser más abundante en la región centro-sur, y 3) no se encontraron rastros del ratón de abazones, sin embargo dado que se encontraron restos de esta subespecie endémica únicamente en las excretas de los perros colectadas en el norte, es posible que esta especie se distribuya exclusivamente en esa zona.

Con este estudio fue posible detectar los patrones de distribución de los perros ferales en la Isla de Cedros e identificar sus principales presas. Los resultados muestran que los perros se alimentan de al menos tres mamíferos endémicos (el venado bura, el conejo matorralero y el ratón de abazones de Cedros) y dos pinnípedos (el elefante marino del norte y el lobo marino de California). Dado que los perros pueden ser depredadores exitosos o de hábitos carroñeros, es necesario realizar estudios de comportamiento alimentario para determinar su eficiencia como depredadores y el nivel de daño asociado sobre las poblaciones de mamíferos nativos. Por otra parte, los resultados sugieren que las interacciones entre pinnípedos y perros son frecuentes, lo que podría representar dos posibles amenazas potenciales. Primero, los disturbios ocasionados por el acoso de los perros podrían desplazar al mediano plazo a los pinnípedos fuera de sus ambientes naturales, provocando el abandono de las colonias. Segundo, dado que los pinnípedos y los perros están relacionado filogenéticamente (Higdon et al. 2007), tal vez la mayor amenaza sea la propagación de enfermedades infecciosas, con serias repercusiones para la vida silvestre.

Agradecimientos. El trabajo de campo fue financiado parcialmente por la fundación Idea Wild y la autora contó con beca postdoctoral otorgada por el CONACyT. La Sociedad Cooperativa de Producción Pesquera "Pescadores Nacionales de Abulón" de la Isla de Cedros brindó apoyo logístico durante el trabajo de campo. El estudio se realizó en colaboración con la dirección del ANP "Valle de los Cirios" y con permiso del Gobierno de México (Of. No. SGPA/DGVS/00340/10).

\section{LITERATURA CITADA}

Brower, J. E. \& J. H. Zar. 1984. Field and laboratory methods for general ecology. William C. Brown Publ., Dubuque.

Caughley, G. 1977. Analysis of vertebrate populations. John Wiley and Sons, London.

Cavalli, P. 1994. Faeces count as an index of abundance. Acta Theriologica, 39: 417-424.

Corbett, L. K. 2001. The dingo in Australia and Asia. J. B. Books Pty. Ltd., Marleston.

De Luca, D. W. \& N. E. Mpunga. 2005. Carnivores of the Udzungwa Mountains: presence, distribution, and threats. Wildlife Conservation Society, Mbeya.

Engeman, R. M. \& L. Allen. 2000. Overview of a passive tracking index for monitoring wild canids and associated species. Integrated Pest Management Reviews, 5: 197-203.

Engeman, R. M. \& G. W. Witmer. 2000. IPN strategies: indexing difficult to monitor populations of pest species. Pp. 183-189. In: T. P. Salmon and A. C. Crabb (eds). Memories Proceedings $19^{\text {th }}$ Vertebrate Pest Conference. University of California Davis, San Diego. 
Fleming, P., L. Corbett, R. Harden \& P. Thomson. 2001. Managing the impacts of dingoes and other wild dogs. Bureau of Rural Resources, Canberra.

Furtado, M. M., S. E. Carrillo-Percastegui, A. T. A. Jácomo, G. Powell, L. Silveira, C. Vynne \& R. Sollmann. 2008. Studying jaguars in the wild: past experiences and future perspectives. Cats News Special Issue, 4: 41-47.

Gallo-Reynoso, J. P. \& M. C. García-Aguilar. 2008. Análisis preliminar de la presencia de perros ferales en la Isla de Cedros, Baja California. Revista Mexicana de Mastozoología, 12: 130-140.

Green, J. S. \& P. S. Gipson. 1994. Feral dogs, pp. 77-81. In: S. E. Hygnstrom, R. M. Timm and G. E. Larson (Eds.). Prevention and control of wildlife damage. University of Nebraska Cooperative Extension Service, Lincoln.

Higdon, J. W., O. R. P Bininda-Emonds, R. M. D. Beck \& S. H. Ferguson. 2007. Phylogeny and divergence of the pinnipeds (Carnivora: Mammalia) assessed using a multigene dataset. Evolutionary Biology, 7: 216-235.

Jachmann, H. 1991. Evaluation of four survey methods for estimating elephant densities. African Journal of Ecology, 29: 188-195.

Karanth, K. U., J. D. Nichols, J. Seidensticker, E. Dinerstein, J. L. Smith, C. McDougal, A. J. T. Johnsingh, R. S. Chundawat \& V. Thapar. 2003. Science deficiency in conservation practice: the monitoring of tiger populations in India. Animal Conservation, 6: 1-10.

Kay, C. E. 1998. Are ecosystems structured from top-down or bottom-up: a new look at an old debate. Wildlife Society B, 26: 484-498.

Khorozyan, I. 2003. Camera photo-trapping of the endangered leopards (Panthera pardus) in Armenia: a key element of species status assessment. Report for the People's Trust for Endangered Species, U. K.

Lowry, M. S. \& O. M. Maravilla-Chávez. 2005. Recent abundance of California sea lions in western Baja California, Mexico and the United States, pp. 485-498. In: D. K. Garcelon \& C. A. Schwenn (Eds.). Proceedings of the $6^{\text {th }}$ California Islands Symposium. National Park Service Technical Publication CHIS-05-01. Institute for Wildlife Studies, Arcata.

Maher, D. S. \& J. R. Brady. 1986. Foodhabits of bobcat in Florida. Journal of Mammalogy, 67: 133138.

Mellink, E. 1993. Biological conservation of Isla de Cedros, Baja California, México: assessing multiple treats. Biodiversity and Conservation, 2: 62-69.

Mitchell, B. \& S. Balogh, S. 2007. Monitoring techniques for vertebrate pest: wild dogs. NSW Department of Primary Industries/Bureau of Rural Science, Canberra.

Mitchell, B. \& P. B. Banks. 2005. Do wild dogs exclude foxes? Evidence for competition from dietary and spatial overlap in the Greater Blue Mountains region of New South Wales. Austral Ecology, 30: $582-591$.

Moore, T. D., L. E. Spence \& C. E. Dugnolle. 1974. Identification of the dorsal guard hairs of some mammals of Wyoming. Wyoming Game and Fish Department. Department Bulletin 14. Cheyenne, Wyoming.

Morán, R. 1972. Las plantas vasculares de Isla de Cedros. Calafia, 2: 34-36.

Reynolds, J. C. \& N. J. Aebischer. 1991. Comparison and quantification of carnivore diet by faecal analysis: a critique, with recommendations, based on a study of the fox Vulpes vulpes. Mammal Review, 21: 97-122.

Rodríguez de la Gala, S. 2002. Catálogo del pelo de guardia de los mamíferos del Estado de Baja California, México. Tesis de Licenciatura, Facultad de Ciencias, UNAM, México D. F.

Rzedowski, J. 1978. Vegetación de México. Limusa, México D. F. 
Sadler, L. M. J., C. C. Webbon, P. J. Baker \& S. Harris. 2004. Methods of monitoring red foxes Vulpes vulpes and badgers Meles meles: are field signs the answer? Mammal Review, 34: 75-98.

Sharp, A., M. Norton, A. Marks \& K. Holmes. 2001. An evaluation of two indices of red fox (Vulpes vulpes) abundance in an arid environment. Wildlife Research, 28: 419-424.

Smith, D. W., R. O. Peterson \& D. H. Houston. 2003. Yellowstone after wolves. Bioscience, 53: 330-340.

Stewart, B. S., P. K. Yochem, H. R. Huber, R. L. De Long, R. J. Jameson, W. J. Sydeman, S. G. Allen \& B. J. Le Boeuf. 1994. History and present status of the northern elephant seals population, pp. 29-48 In: B. J. Le Boeuf and R. M. Laws (Eds.). Elephant seals: population ecology, behavior and physiology. University of California Press, Los Angeles.

Sutherland, W. J. 1996. Mammals, pp: 260-280. In: W. J. Sutherland (Ed.). Ecological census techniques: a handbook. Cambridge University Press, Cambridge.

Terrink, B. J. 1991. Atlas and identification key: hair of west-European mammals. Cambridge University Press, Cambridge.

Wasser, S. K., B. Davenport, E. R. Ramage, K. E. Hunt, M. Parker, C. Clarke \& G. Stenhouse. 2004. Scat detection dogs in wildlife research and management: applications to grizzly and black bears in the Yellowhead ecosystem, Alberta, Canada. Canadian Journal of Zoology, 82: 475-492.

Webbon, C. C., P. H. Baker \& S. Harris. 2004. Faecal density counts for monitoring changes in red fox numbers in rural Britain. Journal of Applied Ecology, 41: 768-779.

Wilson, G. J. \& R. J. Delahay. 2001. A review of methods to estimate the abundance of terrestrial carnivores using field signs and observation. Wildlife Research, 28: 151-164.

Zar, J. H. 1996. Biostatistical analysis. Prentice Hall, New Jersey. 